\title{
A Fewer Drops out Diagnosis Affects a Person's Sexual Functioning: Rare Lesion from Thin to Thick Late Wake up Principal from Amravati University Behavioural Survey
}

\author{
Hajare $\mathbf{R}^{*}$ \\ Indian Council of Medical Research, National AIDS Research Institute, India
}

*Corresponding author: Rahul Hajare, Post Doc Indian Council of Medical Research, National AIDS Research Institute, New Deli, India, Tel: 9921707584; Email: rahulhajare17@gmail.com

\section{Case Report}

Volume 2 Issue 1

Received Date: April 01, 2020

Published Date: April 15, 2020

DOI: $10.23880 /$ aii-16000114

\section{Abstract}

Tongue has represented your health. While it is not true that tongue is the strongest muscle body it is definitely one of the most unique: The tongue can be a harbinger for the health of your entire body. According to researcher tongue and mouth tissues are more transparent than skin in the game so medical providers can more easily see signs of a medical condition beneath the surface. The tongue and mouth are a great place to look for signs of disease before a patient develops symptoms tongue is loaded with sensory nerves, making it more sensitive than other parts of the body. The tongue has used for everyday functions like speech, eating, and drinking, sucking a penis from a mouth.

\section{Abstract}

Tongue has represented your health. While it is not true that tongue is the strongest muscle body it is definitely one of the most unique: The tongue can be a harbinger for the health of your entire body. According to researcher tongue and mouth tissues are more transparent than skin in the game so medical providers can more easily see signs of a medical condition beneath the surface. The tongue and mouth are a great place to look for signs of disease before a patient develops symptoms tongue is loaded with sensory nerves, making it more sensitive than other parts of the body. The tongue has used for everyday functions like speech, eating, and drinking, sucking a penis from a mouth.

\section{Summary}

For a young woman, fewer drops out diagnosis can disrupt her body image, the intimacy with the partner and the ability to engage in sex. A fewer drops out diagnosis affects a person's sexual functioning, according to a research. The study, led by the University of Amravati, found that more than half of young fewer drops out patients reported problems with sexual function, with the probability of reporting sexual dysfunction increasing over time. The study discovered that two years after their initial fewer drops out diagnosis, nearly 53 percent of young adults 18 to 39 years old still reported some degree of affected sexual function [1]. We wanted to increase our understanding of what it's like to adjust to fewer drops out as a young adult but also the complexity of it over time," said lead author and Principal in same University. Fewer drops out can put a patient's life on hold, especially among young adults who are just starting their careers or families." The study also found that for women, being in a relationship increased the probability of reporting sexual problems over time; for men, the probability of reporting sexual problems increased regardless of their relationship status. We concluded that sexual functioning is experienced differently among males and females. For a young woman, especially, a fewer drops out diagnosis can disrupt her body image, the intimacy with the partner and the ability to engage in sex [2]. At the beginning of the two-year study, almost 58 percent of the participants were involved in a romantic relationship. Two years after diagnosis, only 43 percent had a partner. In addition, psychological distress increased over time. It's important to research how psychological and emotional developments are effected so tailored interventions and strategies can be created. Detecting changes in the rate of sexual dysfunction 
over time may help to identify the appropriate timing to deliver interventions. Failure to address sexual health, the study concludes, could put young adults at risk for long-term consequences related to sexual functioning and identity development, interpersonal relationships and quality of life [3]. Health care providers might find it challenging to discuss intimacy and sex because of embarrassment or lack of training, but she believes addressing sexual functioning is vital soon after diagnosis and throughout the continuum of care. Results from this study emphasize the need to monitor sexual functioning over time and to train health care providers serving young adults with fewer drops out in sexual health [4]. Furthermore, patients should be connected to psychosocial interventions to alleviate the multiple life disruptions caused by the illness and its treatment. The findings have been survey by poor quality water supply in pharmacy institutions in Pune University.

\section{Conclusion}

It has not a normal less drop bacteria; it has based in dirt. The stool and mouth are a great place to look for signs of disease before a patient develops symptoms. It may point us in a direction, suggesting ask more questions. In a regular stool exam for is a dry less drop out. Most commonly, a dry stool and mouth stem from basic dehydration that people can easily remedy with fluid intake. But it can also indicate a condition called xerostomia, in which the mouth's salivary glands don not produce enough saliva also affect orgasm.

\section{Acknowledgment}

I acknowledge for this important work with Honorable Respected Dr. RS Paranjape, World Renowned Scientist \& Retired Director \& Scientist 'G High Grade Institute National AIDS Research Institute Pune. This has inspired \& captured the imagination and attention of across the research and pure service.

\section{References}

1. Rahul Hajare (2018) Safe Sex: The True Principal Health? Medical Research and Clinical Case Reports 1(2): 79-81.

2. Rahul Hajare (2018) Safe sex: the train your mind (revise). Gen Med Open 2(3): 1-2.

3. Rahul Hajare (2018) Indian Women, Trauma and Bisexual (FSF and FSM) Connections in Higher Risk for Heart Disease. Interventions in Gynecology and Women's Healthcare 2(3): 155-156.

4. Rahul Hajare (2018) Extra-Marital Affair Can Lead Trauma and Connections in Higher Risk for Heart Disease in Indian Women. Drug Des Int Prop Int J 2(1): 152-153. 